\title{
Efecto del Clima Psicosocial del Grupo y de la Personalidad en el Síndrome de Quemado en el Trabajo de los docentes
}

\author{
Ivette Margarita Espinoza-Díaz*, Jordi Tous-Pallarès, y Andreu Vigil-Colet
}

Universitat Rovira i Virgili, Centre de Recerca en Avaluació i Mesura de la Conducta

\begin{abstract}
Resumen: El presente estudio evalúa las relaciones de la percepción del clima psicosocial y de la personalidad con el síndrome de quemado en el trabajo (SQT) o burnout en docentes de educación infantil, primaria y secundaria con este fin se administraron cuestionarios para evaluar el clima psicosocial, la personalidad y el burnout a una muestra de 386 docentes. Se analizaron las relaciones entre dichas variables y se analizó hasta qué punto las variables de clima y personalidad permiten establecer un modelo predictivo del SQT mediante el análisis de regresión múltiple. Los resultados mostraron que tanto el clima psicosocial (especialmente el grado de desorganización) como la personalidad (especialmente la amabilidad) influyen en el burnout y, aunque los factores de clima parecen ser mejores predictores del SQT, la utilización de medidas de personalidad incrementan la capacidad predictiva de dicho síndrome. De este modo la utilización conjunta de estos dos tipos de predictores mejoran la capacidad de establecer modelos que permitan la prevención del SQT en entornos docentes.

Palabras clave: Clima psicosocial; personalidad; SQT (burnout).
\end{abstract}

\section{Introducción}

La salud ocupacional de los docentes y de los futuros profesionales de la educación es un factor que interviene en el proceso de enseñanza-aprendizaje y que puede afectar a la calidad educativa que ofrece el sistema. (Espinoza-Díaz y Tous-Pallarès, 2011). Los frecuentes cambios asociados al rol del docente, como por ejemplo los propiciados por las reformas educativas, pueden generar un alto grado de malestar debido a que éstos no perciben los recursos suficientes para hacer frente a nuevas demandas, viéndose esto agravado por que cada reforma considera la necesidad de modificar la manera actual de proceder, justificándose bajo el concepto de "dar respuesta a necesidades educativas específicas y a la diversidad” (Lorente, Salanova, Martinez y Schaufeli, 2008).

Las condiciones de trabajo de los profesionales de la educación tienen una influencia significativa sobre su calidad de vida laboral y sobre la calidad del servicio que ofrecen. Así por ejemplo se ha visto que la presencia de demandas específicas y la ausencia de recursos específicos necesarios para hacerles frente generan burnout, lo que tiene consecuencias negativas como la rotación, el absentismo o la reducción del compromiso del docente con la organización (Gil-Monte, Carlotto y Gonçalves, 2011; Maslach, Jackson y Leiter 1996; Moreno-Jiménez, Nelson, Natera, RodríguezMuñoz y Morante, 2006; Tous-Pallarès, 2009).

En este mismo sentido, Lorente et al. (2008), señalan que las demandas del trabajo son un importante predictor del

* Dirección para correspondencia [Correspondence address]:

Ivette Margarita Espinoza-Díaz. Universitat Rovira i Virgili, Centre de Recerca en Avaluació i Mesura de la Conducta. Carretera de Valls s/n, 43007, Tarragona (España).

E-mail: ivettemargarita.espinoza@estudiants.urv.cat

\begin{abstract}
Title: How psychosocial group climate and personality affects teacher burnout.

Abstract: The present study evaluates how psychosocial group climate and personality affects burnout syndrome (BS) in teachers of infant, primary and secondary education. To do so questionnaires were administered to evaluate psychosocial climate, personality and burnout in a sample of 386 teachers. The study analyzes the relation between these variables and the extent to which the variables of climate and personality allow a predictive model of burnout to be established by means of multiple regression analysis. The results showed that both psychosocial climate (particularly the degree of disorganization) and personality (particularly emotional stability) influence burnout and, although climate factors seem to be better predictors of BS, the use of personality measurements increases the predictive capacity of the syndrome. Therefore, the joint use of these types of predictor increases our ability to establish models that can prevent BS in teaching environments.
\end{abstract}

Key words: Psychosocial climate; personality; BS (burnout syndrome).

burnout en docentes, y consideran necesario incluir, además de las variables contextuales, el estudio de los factores de personalidad en su predicción, ya que los recursos personales permiten a los individuos abordar y hacer frente a las demandas externas / internas en situaciones de estrés (Durán, Extremera, Rey, Fernández-Berrocal y Montalbán, 2006; García-Izquierdo, Ramos-Villagrasa y GarcíaIzquierdo, 2009).

En los últimos años el enfoque de la psicología positiva se ha extendido al campo de la Psicología del trabajo y la Salud Ocupacional (Avey, Luthans, Smith y Palmer, 2010; Baker, Rodriguez-Muñoz y Derks, 2012; Rodriguez-Carvajal, Moreno-Jiménez, De Rivas, Bejarano y Sanz-Vergel, 2010; Moreno-Jiménez, Garrosa, Corso, Boada y RodriguezCarvajal, 2012). Desde esta aproximación cabe mencionar que el campo del malestar ocupacional ha sido uno de los tópicos más estudiados, con especial énfasis en sus diferentes manifestaciones asociadas como por ejemplo el estrés, el burnout y la insatisfacción laboral (Otero, Castro, Santiago y Villardefrancos, 2010).

En el ámbito educativo se han realizado diversos trabajos de investigación que analizan el estrés en docentes, en los que se menciona la necesidad de la prevención de daños ocasionados por el estrés derivado por factores psicosociales inherentes a las condiciones de trabajo (Da Silva, Gil-Monte, de Fátima, y Bovi, 2013; Figueiredo-Ferraz, Gil-Monte, Ribeiro, y Grau-Alberola, 2013; Gil-Monte, Carlotto, y Gonçalves, 2011; Gil-Monte, y Noyola, 2011; Gil-Monte, Unda y Sandoval, 2009; Gil-Monte, 2000, 2001, 2011; Mercado-Salas y Gil-Monte, 2012; Mercado-Salgado y Gil-Monte 2010; Pando-Moreno, Aranda-Beltrán, Aldrete-Rodriguez, Flores-Salinas, Pozos-Radillo, 2006; Otero et al. 2010; Unda, 2010; Wang, Zhang y Jackson, 2013). No obstante, la mayoría de estos trabajos no tienen en cuenta el efecto que pue- 
den tener las diferencias individuales en personalidad sobre este tipo de procesos. Hay que tener en cuenta que autores como Egan, Tannahill, Petticrew y Thomas, (2008) plantean la hipótesis de que las respuestas a los factores de clima psicosocial en el grupo de trabajo no son las mismas en todos los trabajadores aunque ocupen un mismo puesto. La personalidad podría influir en la forma de razonar y dar respuesta de los individuos y éstas podrían enfocarse mejor a determinados problemas si éstos concuerdan con su perfil de personalidad (Fumero, Santamaría y Johnson-Laird, 2010; Garcia-Villamisar y Freixas-Guinjoan, 2003).

La influencia del clima psicosocial del grupo y la personalidad sobre la calidad de vida laboral, ha sido documentada desde hace tiempo en los trabajos de Furham (2005) o de Ones y Viswesvaran (2001), que incorporan los "Criterion-focused Occupational Personality Scales (COPS)" para explicar la relación entre clima psicosocial y medidas de personalidad basadas en el modelo de los "cinco grandes" con las características grupales del puesto y con las conductas en el trabajo. En este sentido García-Izquierdo, et al. (2009) citando a Salgado (1998), señalan que los individuos más propensos a sufrir burnout se caracterizan por manifestar ansiedad, niveles bajos de actividad y de control emocional, del mismo modo se les dificulta establecer los límites en las relaciones profesionales lo que conecta con el modelo de personalidad de los Big Five.

La percepción que realiza un empleado del clima del grupo de trabajo derivado de las relaciones profesionales, es clave para la evaluación de su salud psicosocial y calidad de vida de su empleo (Perry, Le May, Rodway, Tracy, y Galer, 2005), si la valoración final de su percepción conduce a daño, pérdida, amenaza o desafío emergerá una respuesta de estrés y consecuencias psicosociales, que en alto grado también pueden ocasionar la aparición del síndrome de burnout (Carvana 2001).

Gil-Monte (2011) define el burnout o síndrome de quemarse en el trabajo (SQT) como

un proceso que se desarrolla de manera progresiva debido a la utilización de estrategias de afrontamiento poco funcionales con las que los profesionales intentan protegerse del estrés laboral generado por sus relaciones con los clientes de la organización y, en menor medida por sus relaciones con la organización (p. 13).

Por lo tanto podemos decir que el SQT, se producirá como una respuesta prolongada a los estresores crónicos de naturaleza emocional e interpersonal (Grau-Arberola, GilMonte, García-Juesas y Figueredo-Ferraz, 2010).

Se considera que el SQT en el docente surge de una discrepancia entre sus ideales individuales orientados a dar una buena formación a sus alumnos y la realidad de las actividades diarias, se centra más en la entrega de informes, avances programáticos, calificaciones, firmas de asistencia, etc., que en dar apoyo (aunque sea emocional) a la labor sustantiva del docente (Pando-Moreno, et al. 2006). De igual manera, las actitudes que desarrollan los profesionales hacia los usuarios de la organización y el servicio que ofrecen están relacionados con riesgos laborales asociados a su entorno, siendo de especial relevancia en el sector asistencial los riesgos laborales de origen psicosocial debido a que los profesionales trabajan en continua interacción con personas. $\mathrm{Y}$ es en la tarea del docente donde es más probable su incidencia, ya que los profesionales del sector educación trabajan con y de cara a las personas desempeñando un importante papel para la sociedad (Oberle y Hughes, 2001 y Ben-Ari, Krole y Har-Even, 2003).

Además, resulta interesante ver como esta constante interacción no les deja exentos de situaciones conflictivas en el grupo de trabajo, Mercado, et al. (2010) argumentan que cuando existen relaciones interpersonales conflictivas los individuos más comprometidos con la organización presentan mayores niveles de SQT que los individuos menos comprometidos especialmente, en las dimensiones de desgaste psiquico y culpa. Como consecuencia si los docentes se sienten descontentos consigo mismos e insatisfechos con sus resultados laborales mostrarán indicadores de agotamiento emocional que posiblemente les llevará a cuestionar su eficacia y a pensar que ya no pueden dar más de sí mismos (Durán, et al. 2006; Gil-Monte y Peiró, 1997; Gil-Monte, Peiró y Valcárcel, 1998; Queirós, Carlotto, Kaiseler, Dias y Pereira, 2013).

Por lo tanto, en su ejercicio profesional, los docentes puede que perciban mayores obstáculos para la realización de sus tareas y que éstos repercutan en unos mayores niveles de SQT en comparación con la población que realiza otras tareas, y como consecuencia, podría verse reflejado en menores niveles de autoeficacia (Salanova, Llorens y GarcíaRenedo, 2003). En este mismo sentido cabe mencionar que dentro de los riesgos laborales de carácter psicosocial, el estrés laboral y SQT ocupan un lugar destacado, y puede afectar a todas las profesiones asistenciales, pero especialmente a dos: la docencia y la asistencia sanitaria, mientras mayor sea la demanda del trabajo y más alto en nivel vocacional del especialista existe mayor probabilidad de que aparezca el síndrome, y esto representa una de las principales causas del deterioro de las condiciones de trabajo, y fuente de absentismo laboral. (Cherniss, (1995); Gil-Monte, 2011; Grau-Alberola, et al. (2010); Moreno-Jiménez, BustosRodriguez, Matallana-Alvarez, y Millanes-Caballero, 1997; Otero et al. 2010; Pando-Moreno, et al. 2006; Tous-Pallarès, 2009; Wang, et al. 2013).

Teniendo en cuenta lo expuesto anteriormente, nuestro objetivo es estudiar la influencia que tiene el clima grupal y la personalidad en la aparición del SQT en los docentes como parte del grupo de profesiones asistenciales, partiendo de las siguientes premisas:

Por un lado consideramos que aquellos profesores en cuyo entorno laboral perciben desorganización y un mayor número de situaciones estresantes (alumnos problemáticos en clase, poco apoyo de los compañeros de trabajo, problemas técnicos en el centro, pérdida de control de la tarea y conflicto de rol, etc.) son los que manifiestarán 
actitudes más cínicas o distantes hacia el trabajo y se sentirán menos competentes en el desempeño de su rol como docente. En este sentido, estudios como el de MorenoJiménez et al. (2012) encontraron correlaciones de $(r=.550$; $p<.01)$ entre el estrés asociado al rol con el agotamiento emocional. En el mismo sentido, autores como Durán et al. (2006), encuentran que la percepción de estrés incrementa el cansancio emocional y el cinismo $(r=.330 ; p<.01)(r=.370$; $p<.01)$ y disminuye la dedicación $(r=-.170 ; p<.01)$, mientras que en los estudios Moreno-Jiménez et al. (2006) se aprecia una relación significativa entre los antecedentes organizacionales con el burnout $(r=.69 ; p<.01)$.

Por otro lado creemos que los factores de personalidad podrían influir en la capacidad del docente para hacer frente y dar respuesta a las condiciones estresantes derivadas de su trabajo actuando estos bien como protectores, o bien como factores que agravan los efectos del SQT. En este sentido, autores como García-Izquierdo et al. (2009) relacionan agotamiento emocional con los Big Five obteniendo las siguientes puntuaciones: con neuroticismo (polo negativo de la estabilidad emocional $)(r=.360 ; p<.01)$, con extraversión $(r$ $=-.150 ; p<.01)$, para apertura a la experiencia $(r=-.180 ; p<$ $.01)$, amabilidad $(r=-.220 ; p<.01)$ y para responsabilidad $(r$ $=-.190 ; p<.01)$. En este mismo sentido Queirós et al. (2013) por su parte relacionan la satisfacción laboral con el cansancio emocional $(r=-.479 ; p<.01)$ y la despersonalización $(r=-.269 ; p<.01)$. Todo ello nos hace hipotetizar la existencia de un patrón de relaciones negativo al relacionar las dimensiones de personalidad con el SQT.

En resumen consideramos que el agotamiento más o menos rápido de los recursos emocionales propios del profesional de la docencia estará mediado por las diferencias individuales derivadas de su personalidad e influida por el clima percibido en su entorno grupal, esta propuesta es coherente con los hallazgos de autores como Chico (2000) que relaciona la intensidad emocional negativa con la extraversión $(r=-.190 ; p<.01)$ y el neurotiscismo $(r=-.580 ; p<.01)$. No obstante, aunque existen diversos estudios que relacionan por separado clima y personalidad con burnout, existen pocos que las analizan de forma simultánea y, aún menos, que analicen dichas relaciones desde el modelo de los cinco grandes factores de personalidad. Por todo ello consideramos que resulta importante explorar, en primer lugar, como se relaciona la percepción del clima psicosocial del grupo y la personalidad con el SQT y, en segundo lugar, analizar la contribución del clima psicosocial y de la personalidad en un modelo predictivo del SQT.

\section{Método}

\section{Participantes}

El estudio se llevó a cabo con una muestra no probabilística cuya tasa de respuesta fue del $99 \%$. Los participantes fueron 386 docentes en activo de 5 centros de Tarragona ciudad. Un $71.5 \%$ de los docentes son mujeres y un $28.5 \%$ hombres con edades comprendidas entre los 18 y los 64 años $(M=31, D T=10.94)$. El grupo de docentes fue integrado por profesores de educación infantil $(23.6 \%)$, primaria $(42.5 \%)$ y secundaria $(33.9 \%)$, de los cuales el $78.9 \%$ trabajan a jornada completa, el $11.5 \%$ a media jornada y el $9.6 \%$ trabaja por horas.

\section{Instrumentos}

ECPT (Escalas de Clima Psicosocial en el Trabajo): Se utilizaron las escalas desarrolladas por Tous-Pallarès et al. (2011). La ECPT es un cuestionario breve que consta de 16 ítems y tres escalas, permite un diagnóstico de los factores psicosociales que los trabajadores perciben como negativos o positivos en sus organizaciones. Las tres escalas que evalúa son: Contenido del trabajo (CT) $(\alpha=.95)$ que evalúa cómo el trabajador emplea sus habilidades y competencias en el puesto, si es responsable sobre las tareas y si tiene capacidad de detectar los estímulos estresantes de su trabajo; Relaciones personales $(\mathrm{RP})(\alpha=.90)$ que valora las relaciones intergrupales y las repercusiones de éstas en la coordinación de los grupos o los equipos de trabajo; y Definición de rol (desorganización) (DR) $(\alpha=.89) \mathrm{da}$ información sobre la percepción que tiene el individuo con la estructura formal de la organización y como ésta le permite afrontar cambios y tener expectativas de crecimiento en la misma.

OPERAS (Overall Personality Assessment Scale). Esta escala fue desarrollada por Vigil-Colet, Morales-Vives, Camps, Tous, y Lorenzo-Seva (2013) y se trata de un test de 40 ítems basado en el modelo de los cinco factores de personalidad que se caracteriza por proporcionar puntuaciones libres de los efectos de aquiescencia y deseabilidad social. La escala presenta una buena validez convergente con otros test ampliamente utilizados dentro del modelo de los cinco grandes siendo la fiabilidad factorial de las escalas de $\left(r_{\theta \theta}=.86\right)$ para extraversión (EX), $\left(r_{\theta \theta}=.77\right)$ para la responsabilidad $(\mathrm{CO}),\left(r_{\theta \theta}=.86\right)$ para la estabilidad emocional (EE), $\left(r_{\theta \theta}=.71\right)$ para amabilidad $(\mathrm{A})$ y $\left(r_{\theta \theta}=.81\right)$ para la apertura a la experiencia (OP).

CESQT (Cuestionario para la Evaluación del Sindrome de Quemado en el trabajo) es un cuestionario elaborado por GilMonte, (2011). El CESQT evalúa las cogniciones, emociones y actitudes de la persona relacionadas con sus experiencias laborales, este test consta de 20 ítems y abarca 4 dimensiones y una escala general: Ilusión por el trabajo (IT) $(\alpha=.90)$ que se define como el deseo del individuo de alcanzar las metas laborales porque suponen una fuente de realización personal; Desgaste psíquico (DP) $(\alpha=.85)$ mide la aparición de agotamiento emocional y físico debido a que en el trabajo se tiene que tratar a diario con personas que presentan o causan problemas; Indolencia (IN) $(\alpha=.74)$ se define como la presencia de actitudes negativas de indiferencia y cinismo hacia los clientes de la organización; Culpa (C) ( $\alpha$ $=.82$ ) hace referencia a la aparición de sentimientos de culpa por el comportamiento y las actitudes negativas en el trabajo; por último la escala general de SQT $(\alpha=.85)$, es el resultado 
de promediar el resultado de las escalas Ilusión por el trabajo, Desgaste psíquico e Indolencia.

Los análisis estadísticos incluidos en esta investigación se llevaron a cabo utilizando el programa SPSS 20.0.

\section{Procedimiento}

Primeramente se contactó con la delegación de la consejería de Educación de la Generalitat de Catalunya con el fin de que convocara a los directores de los centros educativos de la demarcación para llevar a cabo una reunión explicativa del proyecto y de este modo concertar citas y hacer un convenio con los centros interesados (un total de 5) para posteriormente aplicar la batería de test a sus docentes. Los tests se administraron de forma totalmente anónima y voluntaria. Los docentes fueron convocados en un salón de reuniones donde se les proporcionó la información sobre los objetivos de la evaluación psicosocial resaltando la importancia y necesidad de dar una respuesta sincera a los cuestionarios. De igual manera se explicó a cada grupo de docentes que el acuerdo con el centro incluye el compromiso de confidencialidad que les asegura el tratamiento y buen uso de la información aportada tal y como se detalla en los formularios utilizados para administrar los tests.

\section{Resultados}

La Tabla 1 muestra las correlaciones de Pearson obtenidas al relacionar las medidas de clima psicosocial y personalidad con las de SQT. Tal y como puede observarse en dicha tabla, tanto las escalas de clima psicosocial como las de personalidad presentan relaciones substanciales con las medidas de SQT, siendo generalmente mayores las obtenidas entre las medidas de clima y las de SQT. En este sentido destaca especialmente la relación entre definición de rol (desorganización) y la puntuación total de SQT $(r=.532 ; p<.01)$, siendo también relevante la relación de la escala global de SQT con la de relaciones personales $(r=-.305 ; p<.01)$ y con contenido del trabajo $(r=-.260 ; p<.01)$. Por lo que refiere a las escalas específicas del ECPT y del CESQT, todas las relaciones fueron significativas con la excepción de la correlación entre contenido del trabajo y culpa, destacando especialmente las relaciones observadas entre las medidas de desorganización con desgaste psicológico $(r=.476 ; p<.01)$, indolencia $(\mathrm{r}=.374 ; p<.01)$ y culpa $(\mathrm{r}=.291 ; p<.01)$, en tanto que para la medida de ilusión por el trabajo la correlación más alta fue con la escala de relaciones personales $(\mathrm{r}=$ $.363 ; p<.01)$.

Todos estos resultados indican que el elemento del clima más que más influye en el SQT, especialmente en el desgaste psicológico, es la percepción del docente de la desorganización en su entorno laboral, mientras que las relaciones personales y la definición de rol en su puesto de trabajo parecen ejercer un cierto papel protector por lo que refiere al SQT.

Por otro lado cabe resaltar que las correlaciones encontradas entre el SQT y las medidas de personalidad también presentan relaciones destacables. En este sentido, con la excepción de apertura a la experiencia, todas las dimensiones de personalidad presentaron relaciones significativas con la escala total SQT, siendo mayores para la estabilidad emocional $(r=-.378 ; p<.01)$ y amabilidad $(r=-.368 ; p<.01)$ que para extraversión $(r=-.285 ; p<.01)$ y responsabilidad $(r=$ .236; $p<.01)$. Como consecuencia, los individuos más estables emocionalmente, amables y extrovertidos tienden a quemarse menos en el trabajo, al igual que los más responsables.

Analizando de forma más detallada las escalas que componen el CESQT, podemos ver que los cinco factores de personalidad se relacionan significativamente con cada una de ellas. En el caso de la ilusión por el trabajo podemos observar una especial relación con estabilidad emocional $(r=$ $.290 ; p<.01)$, extraversión $(r=.272 ; p<.01)$, amabilidad $(r$ $=.262 ; p<.01)$ y responsabilidad $(r=.262 ; p<.01)$, también cabe mencionar la relación con apertura a la experiencia $(r$ $=.127 ; p<.05)$ aun que ésta presenta un valor considerablemente menor. Por su parte, desgaste psicológico lo hace especialmente con estabilidad emocional, amabilidad y extraversión $(r=-.337 ; p<.01, r=-.258 ; p<.01$ y $r=-.245 ; p<$ $.01)$, y en menor medida con responsabilidad $(r=-.191 ; p<$ .01). Para indolencia la relación más importante es con amabilidad $(r=-.326 ; p<.01)$ seguida por estabilidad emocional $(r=-.222 ; p<.01)$, siendo menor la relación con responsabilidad $(r=-.142 ; p<.01)$ y extraversión $(r=-.131 ; p<.01)$. Por último la escala de culpa muestra una mayor relación con estabilidad emocional $(r=-.295 ; p<.01)$ y amabilidad $(r$ $=.225 ; p<.01)$, relacionándose también con responsabilidad $(r=-.173 ; p<.01)$, extraversión $(r=-.132 ; p<.05)$ y apertura a la experiencia $(r=-.134 ; p<.05)$.

Tabla 1. Correlaciones de Clima Psicosocial y Personalidad CESQT.

\begin{tabular}{lccccc}
\hline Correlación de Pearson & IT & DP & IN & C & SQT \\
\hline Contenido del trabajo & .222 & -.195 & -.180 & -.038 & -.260 \\
Relaciones personales & .363 & -.195 & -.160 & -.125 & -.305 \\
Definición de Rol & -340 & .476 & .374 & .291 & .532 \\
(desorganización) & & & & & \\
Extraversión & .272 & -.245 & -.131 & -.132 & -.285 \\
Estabilidad Emocional & .290 & -.337 & -.222 & -.295 & -.378 \\
Responsabilidad & .203 & -.191 & -.142 & -.173 & -.236 \\
Amabilidad & .262 & -.258 & -.326 & -.225 & -.368 \\
Apertura a la experiencia & .127 & .016 & -.124 & -.134 & -.09
\end{tabular}

$p<.01 ; p<.05$

Nota: Ilusión por el trabajo (IT), Desgaste psicológico (DP), Indolencia, cinismo (IN), Culpa (C), Escala total del Síndrome de quemado en el trabajo (SQT).

En resumen, parece que los docentes más estables, amables, extravertidos y responsables tienden a presentar menores niveles de SQT, así como una mayor ilusión por el trabajo, menor desgaste psicológico así como menores niveles de culpa y cinismo.

Una vez visto que, tal y como se esperaba, tanto las variables del clima como las dimensiones de personalidad están relacionadas con el SQT, se llevaron a cabo una serie de regresiones múltiples por pasos sucesivos con la finalidad de 
conocer qué proporción de varianza del CESQT explican las variables de clima social y de personalidad y si su combinación mejora la predicción del SQT, En dichos análisis se utilizaron como criterios la puntuación total del CESQT y la puntuación de las escalas que lo componen y como predictores las escalas del ECPT y de OPERAS. La Tabla 2 muestra los resultados obtenidos en dichos análisis.

Como podemos ver el mejor predictor de la escala total del CESQT es la escala de desorganización que explica más del $28 \%$ de la varianza de la misma. Es destacable señalar que ninguna de las otras dos escalas de clima entran a formar parte del modelo, lo cual indica que la varianza del SQT explicada por las mismas es común a la explicada por la escala de desorganización. A continuación el modelo incorpora todas las escalas de personalidad, con la excepción de apertura a la experiencia. Las variables de personalidad incrementan en un $12.5 \%$ la varianza explicada por el modelo. De este modo los resultados obtenidos señalan que aunque las variables de clima psicosocial presentan un mayor poder predictivo del SQT (especialmente el grado de desorganización), las variables de personalidad incrementan de forma substancial la capacidad predictiva del modelo.

$\mathrm{Al}$ realizar el mismo análisis para cada una de las escalas del SQT vemos como los resultados son similares, siendo la escala de desorganización el mejor predictor de clima psicosocial y estabilidad emocional y extraversión de personalidad en la mayoría de las mismas. Las distintas dimensiones de personalidad aportan un incremento de la varianza explicada, siendo incluso en una escala concreta (culpa) mayor el porcentaje $(10 \%)$ que explican las variables de personalidad que las de clima (6.5\%). Cabe reseñar que las dimensiones de relaciones personales y contenido del trabajo añaden poco o nada al modelo, salvo en el caso de ilusión por el trabajo donde relaciones personales explica el mayor porcentaje de la varianza explicada $(13 \%)$.

Tabla 2. Regresiones lineales por pasos sucesivos de las escalas de Clima Psicosocial y Personalidad sobre las escalas del CESQT (varianza total explicada, incremento de varianza asociado a cada predictor y pendiente)

\begin{tabular}{|c|c|c|c|c|c|c|c|c|c|c|}
\hline & \multicolumn{2}{|c|}{ SQT } & \multicolumn{2}{|c|}{ IT } & \multicolumn{2}{|c|}{ DP } & \multicolumn{2}{|c|}{ IN } & \multicolumn{2}{|c|}{$\mathrm{C}$} \\
\hline & $\Delta \mathrm{R}^{2}$ & $\beta$ & $\Delta R^{2}$ & $\beta$ & $\Delta R^{2}$ & B & $\Delta R^{2}$ & $\beta$ & $\Delta R^{2}$ & $\beta$ \\
\hline Definición de Rol (desorganización) & .283 & .003 & .062 & -.020 & .226 & .051 & .140 & .031 & .051 & .021 \\
\hline Relaciones personales & - & - & .132 & .030 & - & - & - & - & - & - \\
\hline Contenido del trabajo & - & - & - & - & - & - & - & - & .014 & .014 \\
\hline Amabilidad & .029 & -.003 & .013 & .009 & - & - & .059 & -.020 & - & - \\
\hline Estabilidad Emocional & .065 & -.003 & - & - & .052 & -.024 & - & - & .087 & -.025 \\
\hline Responsabilidad & .007 & -.003 & - & - & .010 & -.010 & - & - & .012 & -.009 \\
\hline Extraversión & .024 & -.002 & .029 & .011 & .015 & -.011 & - & - & - & - \\
\hline Apertura a la experiencia & - & - & - & - & .009 & -.005 & - & - & - & - \\
\hline Varianza Total Explicada (\%) & 40.8 & - & 23.6 & - & 31.2 & - & 19.9 & - & 16.4 & - \\
\hline
\end{tabular}

$p<.01 ; p<.05$

Nota: Ilusión por el trabajo (IT), Desgaste psicológico (DP), Indolencia, cinismo (IN), Personalidad con Culpa (C), Escala total del Síndrome de quemado en el trabajo (SQT).

\section{Discusión}

Los resultados expuestos anteriormente van en la línea apuntada en la introducción, en el sentido que el clima organizacional es uno de los principales determinantes del SQT. De este modo, el clima y, especialmente la desorganización organizacional facilitan la aparición del SQT. No obstante, tal y como se había hipotetizado, los resultados apuntan también a que ciertas dimensiones de personalidad parecen actuar como "protectoras" frente a este síndrome, de tal modo que, especialmente los individuos estables emocionalmente, amables y extravertidos parecen tener menores niveles en este síndrome o en alguno de sus componentes, mientras que la responsabilidad y la apertura a la experiencia presentan también un cierto papel protector.

Centrándonos en la influencia que tiene el clima grupal en la aparición del SQT en los docentes habíamos considerado que los docentes en cuyo entorno laboral perciben una mayor desorganización manifiestarán actitudes más cínicas o distantes hacia el trabajo y se sentirán menos competentes en el desempeño de su rol como docente. En este sentido, tal y como suponíamos inicialmente, encontramos que la desorganización influye en el desgaste psicológico y en la indolencia (cinismo) en el mismo sentido que apuntaban los estudios de Moreno-Jiménez et al. (2012) y Durán et al. (2006).

En lo relativo a las dimensiones de personalidad, propusimos que podrían influir en la capacidad del docente para hacer frente y dar respuesta a las condiciones estresantes derivadas de su trabajo, actuando estas como protectores ante el SQT. En este sentido los datos encontrados apoyan también, tal y como esperábamos inicialmente, que las características de la personalidad de los individuos, ejercen una influencia moderadora ante el SQT, incrementando la ilusión por el trabajo, disminuyendo el desgaste psicológico, la indolencia y la culpa. Por otra parte este efecto "protector" lo ejercen especialmente la estabilidad emocional, la extraversión y la amabilidad, y en menor medida la responsabilidad. Estos resultados apoyan parcialmente estudios como los de Queirós et al. (2013), que asocian a la variable de personalidad resistente una relación con satisfacción laboral $(r=-.418$; $p<.01)$ y cansancio emocional $(r=-.326 ; p<.01)$ respectivamente, donde la personalidad resistente se caracteriza por la tendencia a implicarse en las actividades de la vida e iden- 
tificarse con el significado de los eventos. Hay que destacar que diversos estudios han presentado un patrón de relaciones entre la personalidad resistente o resiliente y el agotamiento emocional equivalente al encontrado por nosotros en el presente estudio, es decir, relaciones positivas de la personalidad resiliente y negativas del agotamiento emocional con la estabilidad emocional, la amabilidad, la extraversión y la responsabilidad (Campbell-Sills, Cohan y Stein, 2006; García-Izquierdo, et al. 2009; Maddi, Khoshaba, Persico, Lu, Harvey, y Bleecker, 2002; Zhang, 2011)

Los resultados obtenidos al relacionar las escalas del ECPT y del CESQT señalan que la desorganización organizacional es el elemento del clima psicosocial de mayor incidencia negativa en docentes, facilitando la aparición del SQT, por lo tanto también se presenta como su principal predictor. No obstante, cabe resaltar que a pesar de que la desorganización organizacional parece ser la variable más relevante en el SQT, el papel de la personalidad también es relevante. De este modo, la inclusión de las variables de personalidad en un modelo predictivo del SQT mejora de forma substancial la capacidad del modelo para pronosticar la posible aparición del SQT en los individuos. En este sentido, si bien es cierto que el mejor predictor del SQT es la escala de clima psicosocial "desorganización" explicando el 28\% de la varianza en la escala global del test CESQT, también es destacable que las variables de personalidad incrementan en un $12 \%$ la capacidad predictiva del modelo y, que en algunas escalas particulares del SQT son incluso mejores predictoras que el clima psicosocial. Estos resultados estarían en la línea propuesta por Otero et al. (2010), que en un estudio realizado en una muestra de docentes concluyen que las variables personales, psicosociales y de contexto son las que mejor explican en términos de varianza el SQT. De este modo, el agotamiento más o menos rápido de los recursos emocionales propios del profesional de la docencia estará mediado por las diferencias individuales derivadas de su personalidad e influida por el clima percibido en su entorno grupal.

\section{Referencias}

Avey, J. B., Luthans, F., Smith, R. M., y Palmer, N. F. (2010). Impact of the positive psycological capital on employee well-being over time. Journal of Occupational Health Psycology, 15, 17-28.

Bakker, A. B., Rodríguez-Muñoz, A., y Derks, D. (2012). La emergencia de la Psicología de la Salud Ocupacional Positiva. Psicothema, 24 (1), 66-72.

Ben-Ari, R., Krole R., y Har-Even, D. (2003). Differential effects of simple frontal versus complex teaching strategy on teachers'stress, burnout, and satisfaction. International Journal of Stress Management, 10, 173-195.

Campbell-Sills, L., Cohan, S. L., y Stein, M. (2006). Relationship of resilience to personality, coping, and psychiatric symptoms in young adults. Behaviour Research and Therapy, 44, 585-599.

Carvana, A. (2001). Educación emocional y prevención del estrés en el profesorado. Orientación y Tutoría Intercambio de Experiencias y Recursos. Curso 2000-2001. Cefire Elda, Alicante.

Cherniss, C. (1995). Beyond burnout: helping teachers, nurses, therapists, and lawyers recover from stress and disillusionment. New York: Routledge.

Chico-Librán, E. (2000). Intensidad emocional y su relación con extraversion y neuroticismo. Psicothema, 12, 568-573.
Además de los resultados expuestos anteriormente, cabe señalar que los factores psicosociales negativos relacionados con el contenido del trabajo y las relaciones personales también presentan relaciones relevantes con el clima psicosocial, siendo especialmente alta la relación de la escala de "desorganización" con "desgaste psicológico" e "indolenciacinismo". Este resultado apoya el estudio de Pando et al. (2006), en el que encontraron que los factores psicosociales negativos muestran relaciones significativas con el Agotamiento Emocional (desgaste psicológico) y la Despersonalización (indolencia, cinismo), igual situación se encontró entre los factores psicosociales de tipo Organizacional y las dimensiones del SQT en una muestra de docentes.

Es posible que la influencia que ejerce la percepción de un entorno desorganizado modifique el patrón de comportamiento del grupo de trabajo produciendo desgaste psicológico y llevándoles al grado de la indolencia explicando así en mayor medida el SQT en docentes. Por otro lado, la influencia de los factores de personalidad amabilidad y responsabilidad también es de gran importancia debido a que el papel que desempeñan éstas variables es crucial tanto para predecir como para atenuar la aparición del SQT en docentes.

La presente investigación no está sin embargo exenta de ciertas limitaciones. Así hay que tener en cuenta que se ha centrado en las relaciones entre clima, personalidad y SQT, pero posiblemente existan otros aspectos individuales (p.e. bienestar psicológico y percepción de agresividad en el entorno) o grupales (p.e. liderazgo de equipos docentes y clima) que influyan en el SQT y permitan elaborar un modelo más completo que mejore la predicción de este síndrome. El papel de dichas variables deberá ser contrastado en próximas investigaciones. Asimismo será necesario llevar a cabo investigaciones posteriores que permitan establecer si las relaciones descritas anteriormente son generalizables a cualquier entorno laboral o si por el contrario son debidas a características específicas del ámbito docente.

Da Silva, L., Gil-Monte, P. R., de Fátima, R., y Bovi, G. M. (2013). Prevalência da síndrome de burnout em uma amostra de professores universitários brasileiros. Psicologia: Reflexao e Crítica, 26(4), in press.

Duran. A., Extremera, N., Rey, L., Fernández-Berrocal, P., y Montalbán, M. (2006). Predicting academic burnout and engagement in educational settings: Assessing the incremental validity of perceived emotional intelligence beyond perceived stress and general self-efficacy. Psicothema, 18, 158-164.

Egan, M., Tannihill, C., Petticrew, M., y Thomas, S. (2008). Psychosocial risk factors in home and community settings and their associations with population health and health inequalities: A systematic meta-review. BMC Public Health

Espinoza-Díaz, I. M. y Tous-Pallarès, J. (2011). Calidad de vida y salud ocupacional en organizaciones educativas. Revista del COPC. 229, 46-52.

Figueiredo-Ferraz, H., Gil-Monte, P. R., Ribeiro, B., y Grau-Alberola, E. (2013). Influence of a cognitive behavioural training program on health: A study among primary teachers. Revista de Psicodidáctica, 18(2), 343-356. 
Figueiredo-Ferraz, H., Gil-Monte, P. R., y Grau, E. (2009). Prevalencia del Síndrome de Quemarse por el Trabajo (Burnout) en una muestra de maestros portugueses. Aletheia, 29, 6-15.

Fumero, A., Santamaría, C., y Johnson-Laird, P., (2010). Formas de pensar: efecto de la personalidad en el razonamiento. Psicothema, 22 (1), 57-62.

Furham, A. (2005). Personalidad y diferencias individuales en el trabajo. Madrid: Pirámide.

García-Villamisar y Freixas-Guinjoan, T. (2003). El estrés del Profesorado una perspectiva internacional. P. 26, Ed. Promolibro. Valencia, España.

García-Izquierdo, A. L., Ramos-Villagrasa P. J., y García-Izquierdo, M. (2009) Los Big Five y el Efecto Moderador de la Resistencia en el Agotamiento Emocional. Revista de Psicología del Trabajo y de las Organizaciones, $25,135-147$

Gil-Monte, P. R. (2000). Aproximaciones psicosociales y estudios diagnósticos sobre el síndrome de quemarse por el trabajo (burnout). Monográfico, Revista de Psicología del Trabajo y de las Organizaciones, 16, 101-102.

Gil-Monte P. R. (2001). El Sindrome de Quemarse por el Trabajo (Sindrome de Burnout): aproximaciones teóricas para su explicación y recomendaciones para la intervención. (En línea). España: Universidad de Valencia, Facultad de Psicología, Departamento de Psicología Social y Organizacional. Recuperado de http://www.psicologiacientifica.com/bv/psicologia-78-1-elsindrome-de-quemarse-por-el-trabajo-(síndrome-de-burnout).html

Gil-Monte, P. R., (2011). Cuestionario para la Evaluación del Sindrome de Quemarse por el Trabajo. Tea Ediciones, Madrid, España.

Gil-Monte, P. R., Carlotto, M. S., y Gonçalves, S. (2011). Prevalence of burnout in a sample of Brazilian teachers. European Journal of Psychiatry, 25, 205-212.

Gil-Monte, P. R., y Noyola, V. S. (2011). Estructura factorial del Cuestionario para la Evaluación del Síndrome de Quemarse por el Trabajo en maestros mexicanos de educación primaria. Revista Mexicana de Psicología, $28,75-84$.

Gil-Monte, P. R. y Peiró, J. M. (1997). Desgaste psíquico en el trabajo: el sindrome de quemarse. Madrid: Síntesis.

Gil-Monte, P. R., Peiró, J. M., y Valcárcel, P. (1998). A model of burnout process development: An alternative from appraisal models of stress. Comportamento Organizacional e Gestao, 4, 165-179.

Gil-Monte, P. R., Unda S., y Sandoval J. I. (2009). Validez factorial del "Cuestionario para la Evaluación del Síndrome de Quemarse por el Trabajo" (CESQT) en una muestra de maestros mexicanos. Salud Mental, 32(3), 205-214

Grau-Alberola, E., Gil-Monte, P. R., Garcia-Juesas, J. A., y FigueiredoFerraz, H. (2010). Incidence of burnout in spanish nursing professionals: a longitudinal study. International Journal of Nursing Studies, 47, 10131020

Lorente, L., Salanova, M., Martínez, I., y Schaufeli, W., (2008). Extension of the Job Demands-Resources model in the prediction of burnout and engagement among teachers over time. Psicothema 20 (3), 354-360.

Maddi, S. R., Khoshaba, D. M., Persico, M., Lu, J., Harvey, R., y Bleecker, F. (2002). The personality construct of hardiness, II: Relationships with comprehensive tests of personality and psychopathology. Journal of $R_{\ell-}$ search in Personality, 36, 72-85.

Maslach, C. y Jackson, S. E. (1986). Maslach Burnout Inventory. 2da. Edición, Palo Alto California, Consulting Psychologists Press.

Maslach, C., Jackson, S. E., y Leiter, M. P. (1996). Maslach Burnout Inventory: Manual (3er ed.). Palo Alto, CA: Consulting Psychologist Press.

Management Sciences for Health (2005). Workgroup climate assessment (WCA) tool and guide for facilitators. The Health Manager's Toolkit. Retrieved from http: / / erc.msh.org $/$ mainpage.cfm?file=96.9.htmymodule=toolkitylang uage $=$ English $)$. Boston, MSH.

Mercado-Salas, A. y Gil-Monte, P.R. (2012). Características psicométricas del Cuestionario para la evaluación del síndrome de quemarse por el trabajo en maestros mexicanos. Revista de Educación, 359, 260-273.

Mercado-Salgado, P., y Gil-Monte, P. R. (2010). Influencia del compromiso organizacional en la relación entre conflictos interpersonales y el síndrome de quemarse por el trabajo (burnout) en profesionales de servicios (salud y educación). Innovar, 20(38), 161-174.

Moreno-Jiménez, B., Bustos-Rodriguez, R., Matallana-Alvarez, A., y Miralles-Caballero, T. (1997). La evaluación del burnout, problemas y alter- nativas. El CBB como evaluación de elementos del proceso. Revista de Psicología del Trabajo y de las Organizaciones, 13, 185-207.

Moreno-Jiménez, B., Garrosa, E., Corso, S., Boada, M., y RodriguezCarvajal, R. (2012). Personalidad resistente y capital psicológico: las variables personales positivas y los procesos de agotamiento y vigor. Psicothema, 24, 57-62.

Oberle, K. y Hughes, D. (2000). Doctor's and nurse's perceptions of ethical problems in end-of-life decisions. Journal of Advanced Nursing, 33, 707715.

Moreno-Jiménez, B., Nelson, I., Natera, M., Rodríguez-Muñoz, A., y Morante, M. E. (2006). La personalidad resistente como variable moduladora del síndrome de burnout en una muestra de bomberos. Psicothema, 18, 413-418.

Oberle, K. y Hughes, D. (2001). Doctors' and nurses' perceptions of ethical problems in end-of-life decisions. Journal of advanced nursing, 33, 707-15.

Ones, D. S. y Viswesvaran, C. (2001). Integrity tests and other criterionfocused occupational personality scales (COPS) used in personnel selection. International Journal of Selection and Assessment, 9, 31-39.

Otero, J. M., Castro, C., Santiago, M. J., y Villardefrancos, E. (2010). Exploring Stress, Burnout, and Job Dissatisfaction in Secondary School Teachers. International Journal of Psychology and Psychological Therapy, 10, 107-123.

Pando-Moreno, M., Aranda-Beltrán, C., Aldrete-Rodriguez, M., FloresSalinas, E., y Pozos-Radillo, E. (2006). Factores psicosociales y burnout en docentes del centro universitario de ciencias de la salud. Revista Medigraphic, 8 .

Perry, C., Le May, N., Rodway, G., Tracy, A., y Galer, J. (2005). Validating a work group climate assessment tool for improving the performance of public health organizations. Human Resources for Health, 21, 3-10.

Queiros, C., Carlotto, M.S., Kaiseler, M., Dias, S., y Pereira, A. M. (2013). Predictors of burnout among nurses: An intetactionist approach. Psicothema, 25 (3), 330-335.

Rodríguez-Carvajal, R., Moreno-Jiménez, B., De Rivas, S., Bejarano, A., y Sanz-Vergel, A. I. (2010). Positive psychology at work: Mutual gains for individuals and organizations. Revista de Psicología del Trabajo y de las Organizaciones, 26, 235-253.

Salanova, M., Llorens, S., y García-Renedo, M., (2003). ¿Por qué se están "quemando" los Profesores? INSHT, 28, 16-20.

Salgado, J. (1998). Burnout, Personality and Self-Perceived Job Performance. Paper presented at the XXIV Congress of the International Association of Applied Psychology (IAAP), San Francisco (USA), 9-14 August. Paper presented at the XXIV Congress of the International Association of Applied Psychology (IAAP), San Francisco (USA) 9-14 August.

Salgado, J. F. (2002). The Big Five personality dimensions and counterproductive behaviors. International Journal of Selection and Assessment, 10, $117-$ 125.

Salgado, J. F. (2003). Predicting job performance using FFM and non-FFM personality measures. Journal of Occupational and Organizational Psychology, 76, 323-346.

Tous-Pallarès, J. (2009). El comportamiento absentista. Oviedo: Septem Ediciones.

Tous-Pallarès, J. Bonasa-Jiménez, M.P. Mayor-Sánchez, C., y EspinozaDíaz, I. M., (2011). Escala de Clima Psicosocial en el Trabajo: desarrollo y validación. Anuario de Psicología. 34(1) 214-226.

Unda, S. (2010). Estudio de prevalencia del síndrome de quemarse por el trabajo (SQT) y su asociación con sobrecarga y autoeficacia en maestros de primaria de la Ciudad de México. Ciencia y Trabajo, 35, 257-262.

Vigil-Colet, A., Morales-Vives, F., Camps, E., Tous-Pallarès, J., y LorenzoSeva, U. (2013). Development and validation of the Overall Personality Assessment Scale (OPERAS). Psicothema, 25, 100-106.

Wang, J. L., Zhang, D. J., y Jackson, L. A. (2013), Influence of self-esteem, locus of control, and organizational climate on psychological empowerment in a sample of Chinese teachers. Journal of Applied Social Psychology, 43, 1428-1435

Zhang, L. (2011). Hardiness and the Big Five pe rsonality traits among Chinese university students. Learning and Individual Differences, 21, 109-113.

(Artículo recibido: 10-05-2013; revisado: 20-07-2013; aceptado: 20-01-2014) 